\title{
LA TEORÍA DE LOS GRUPOS DE REFERENCIA*
}

\author{
Cláudio Abreu** \\ Universidad Nacional de Tres de Febrero / Universidad Nacional \\ de Quilmes / ANPCyT (Argentina)
}

\begin{abstract}
Resumen
El objetivo de este artículo es presentar una reconstrucción de la teoría de los grupos de referencia. La teoría, desarrollada por Robert K. Merton, ha sido largamente utilizada en los más variados campos de las ciencias sociales, aunque con escasa o nula consideración metateórica hasta ahora. La reconstrucción atenderá a la presentación que de la misma se recoge en sendos artículos de su libro Social Theory and Social Structure, de 1968, a saber: "Contributions to the theory of reference group behavior" y "Continuities in the theory of reference groups and social structure". Para presentar esta reconstrucción me apoyaré en el instrumental de la Metateoría Estructuralista. Después de una consideración informal de la teoría, serán presentados sus modelos potenciales, modelos, modelos potenciales parciales, sus aplicaciones intencionales y algunos comentarios acerca de la noción de "grupo de referencia" en lo que respeta su carácter relacional y disposicional.
\end{abstract}

Palabras-clave: Teoría de los grupos de referencia, Merton, metateoría estructuralista.

\section{Abstract}

The goal of this article is to present a reconstruction of the reference groups theory. The theory developed by Robert K. Merton is a theory largely used in the most varied fields of

Recibido: 24/02/2011. Aceptado: 16/05/2012.

* Estoy agradecido a la Agencia Nacional de Promoción Científica y Tecnológica de Argentina que, por medio del proyecto de investigación PICT $\mathrm{N}^{\circ} 1558$, me brinda una beca doctoral y financia el proyecto de investigación PICTR2006 No 2007, así como también al CONICET que financia el proyecto PIP N ${ }^{\circ}$ 112-201101-01135, de los cuales participa este trabajo. Agradezco también a los integrantes del Seminario de Filosofía de la Ciencia, en especial a Pablo Lorenzano, Mercedes O’Lery, Santiago Ginnobili y Christián Carman por sus sugerencias en las discusiones sobre las versiones previas. A José L. Falguera le agradezco el constante empeño, los muchos diálogos y sugerencias acerca de la versión final y la ayuda con el español. Ninguno de ellos son responsables de las deficiencias que aún preserve el trabajo.

$\because$ e-mail: claudioabreu@outlook.com 
social sciences but, still up to this day, it does not have a more accurate treatment from the metascientific standpoint. The reconstruction will attend the presentation of it is reflected in two articles in his book Social Theory and Social Structure, 1968, namely: "Contributions to the theory of reference group behavior" and "Continuities in the theory of reference groups and social structure". To present this reconstruction, we will use the instruments of a contemporary conception of theories, namely the Structuralist Metatheory. After an informal account of the theory will be presented their potential models, models, partial potential models, applications intended and some comments about the notion of "reference group" in what respects its relational and dispositional nature.

Keywords: Reference groups theory, Merton, structuralist metatheory.

\section{Introducción}

El objetivo de este artículo es presentar una reconstrucción de la teoría de los grupos de referencia (TGR), la teoría desarrollada por Robert K. Merton. Se trata de una teoría largamente utilizada en los más variados campos de las ciencias sociales, pero que hasta hoy no tuvo un tratamiento más preciso desde el punto de vista metacientífico. En este artículo se trata la TGR tal y como ha sido presentada por Merton en sus célebres artículos integrados en Social Theory and Social Structure, 1968, a saber: "Contributions to the theory of reference group behavior" $\mathrm{y}$ "Continuities in the theory of reference groups and social structure" ${ }^{1}$. El ulterior desarrollo de la teoría será tratado en posteriores trabajos.

Para presentar esta reconstrucción, haremos uso del instrumental de una concepción contemporánea de las teorías, a saber, la Metateoría Estructuralista. Después de una consideración informal de la TGR, serán presentados sus modelos potenciales, modelos, modelos potenciales parciales, sus aplicaciones intencionales y algunos comentarios acerca de la noción de "grupo de referencia" en lo que respeta su carácter relacional y disposicional. De esta manera se puede precisar qué es lo que aporta el trabajo de Merton al entendimiento de la conducta del individuo en sociedad.

Es importante esclarecer que la TGR es un ejemplo de lo que Merton denomina teoría de alcance intermedio, o sea, aquellas teorías que se encuentran a mitad de camino

[...] entre esas hipótesis de trabajo menores pero necesarias que se producen abundantemente durante las diarias rutinas de la investigación y los esfuerzos sistemáticos totalizadores por desarrollar una teoría unificada que explicara todas las uniformi-

${ }^{1}$ Los dos capítulos aparecen en la segunda edición del libro (1957). La primera edición es de (1949). 
dades observadas de la conducta, la organización y los cambios sociales. (Merton, 1968 , p.39 [2002, p. 56]) $)^{2}$

Cada una de ellas, debería así considerarse

[... J una teoría intermedia a las teorías generales de los sistemas sociales que están demasiado lejanas de los tipos particulares de conducta, de organización y del cambio sociales para tomarlas en cuenta en lo que se observa y de las descripciones ordenadamente detalladas de particularidades que no están nada generalizadas. (Merton, 1968, p.39 [2002, p. 56])

Conviene señalar que, a los efectos de la reconstrucción en el marco estructuralista desde una perspectiva sincrónica, la TGR se concibe como un elemento teórico aislado. Ello se debe a que las aportaciones de Merton no hacen referencia a posibles distinciones relevantes que pongan en escena la eventualidad de tener algunas especializaciones. Posibles desarrollos de las aportaciones de Merton, en la línea de desplegar diferentes especializaciones para diferentes grupos de aplicaciones, convertirían lo aquí presentado o en un elemento teórico básico de una red teórica o en un elemento especializado de un elemento teórico básico de alguna teoría de mayor alcance. Como ya se indicó, explorar tales posibilidades queda pospuesto para algún futuro trabajo.

\section{Aclaraciones preliminares}

No obstante, parece oportuno empezar por aclarar que, según Merton, es característico de las teorías de alcance intermedio que dichas teorías "no permanecen separadas, sino que se reúnen en redes más amplias de teorías, como se ilustra con las teorías del nivel de aspiración, grupo de referencia y estructura de oportunidad" (Merton, 1968, p. 68 [2002, p. 97]). Al respecto, tal y como asume explícitamente Merton al principio del segundo de los artículos referentes a la TGR, "Continuities in the theory of reference groups and social structure", "[sus] páginas [...] están organizadas en relación con problemas teóricos, tanto de grupos de referencia como de materias afines a la estructura social en general" (Merton, 1968, p. 335 [2002, p. 362]). Este comentario nos ayuda a entender una afirmación de Merton al final del mismo artículo, cuando escribe acerca de las consecuencias de la conducta relativa a grupos de referencia que:

$\mathrm{Al}$ poner fin a esta exposición sobre continuidades de la teoría de la conducta relativa a grupos de referencia, me limito a mencionar, más bien que a analizar, problemas

\footnotetext{
${ }^{2}$ En adelante, lo puesto entre corchetes corresponde a la versión castellana.
} 
escogidos de las consecuencias de diferentes tipos de conducta relativa a grupo de referencia. Estudiarlos con el detalle que ahora es posible sería convertir esto en todo un libro extenso más bien que en una información provisional. (Merton, 1968, p. 438 [2002, p. 468])

Con independencia de la extensión, parece que las consecuencias a las que se refiere no requieren considerarse necesariamente para identificar lo que es central para la TGR. Además, al tratar específicamente de las funciones y disfunciones de la conducta relativa a un grupo de referencia, dice:

[...] hay 'coherencia entre la teoría de grupo de referencia y ciertos conceptos de la sociología funcional. Parece que éstos se refieren a aspectos diferentes del mismo asunto: uno se enfoca sobre el proceso mediante el cual los individuos se relacionan con los grupos y refieren su conducta a los valores de los grupos; los otros se enfocan sobre las consecuencias de los procesos primordialmente para las estructuras sociales, pero también para los individuos y los grupos comprendidos en las estructuras sociales'. (Merton, 1968, p. 438 [2002, p. 468])

Así tenemos que Merton afirma que la TGR, dicho de modo general, "trata de sistematizar los determinantes y consecuencias de los procesos de valorar y de auto-estimación en que los individuos toman los valores o las normas de otros individuos como sistema comparativo de referencia" (Merton, 1968, p. 228 [2002, p. 314]). Pero además, presenta ideas como la de que "la función de la conformidad es la aceptación por el grupo, así como la progresiva aceptación por el grupo refuerza la tendencia a la conformidad" (Merton, 1968, p. 308 [2002, pp. 334-335]). Aquí, con la reconstrucción que proponemos, tratamos de elucidar lo que concierne a la explicación de la conducta relativa a grupos de referencia. Las cuestiones relativas a 'determinantes', 'consecuencias' y 'función', a las que se hace referencia en las anteriores citas, entendemos que aunque indirectamente tienen que ver con la TGR, no constituyen lo que dicha teoría es directamente en sí misma.

Sin embargo, esta mezcla de problemas y/o perspectivas teóricas presentes en dichos artículos parecen ser una muestra paradigmática de lo que Merton expresa por medio de la noción de 'alcance intermedio', no tanto por lo que se refiere a las teorías de alcance intermedio sino que más bien a la orientación de alcance intermedio, o sea, a la idea de que:

La teoría sociológica, si ha de avanzar de manera significativa, debe proceder sobre estos planes interrelacionados: 1) desarrollando teorías especiales sobre las cuales derivar hipótesis que se puedan investigar empíricamente y 2) desarrollando, no revelando súbitamente, un esquema conceptual progresivamente más general que sea adecuado para consolidar grupos de teorías especiales. (Merton, 1968, p. 51 [2002, p. 68]) 
Esta contextualización hace pensar que Merton entiende su estudio de los grupos de referencia como una oportunidad de sofisticación del funcionalismo. La noción de 'función' (estrechamente vinculada con la noción de 'consecuencias') permite retomar aspectos como el de la conformidad o la inconformidad con, por ejemplo, el intra-grupo. Con ello se estaría apuntando a un vínculo importante con la teoría de la anomia. Lo que hay entonces son relaciones entre 'teorías especiales' en un proceso de 'revelación de un esquema conceptual progresivamente más general'.

Este esquema conceptual, que es progresivamente más general en lo que respecta a la conducta del individuo, involucra, en la obra de Merton, la teoría de la anomia - donde metas culturales sirven de guía a la conducta del individuo- y la teoría de los grupos de referencia - donde los valores de un grupo de referencia también sirven de guía a la conducta del individuo-. En ambas teorías, aunque la referencia es externa (social), encontramos que la práctica creativa de los individuos es significativamente importante para las mismas. En este contexto, por ejemplo, a partir de la noción de grupo de referencia, Merton trabaja nociones como 'integridad', 'visibilidad' y 'autoridad' de los grupos sociales, de manera tal que con estas nociones puede tratar sobre la retroalimentación de un sistema social, llegando así a teorías descriptivo-explicativas de la producción y la manutención del sistema social. Con eso, hace que el funcionalismo sea capaz de explicar, haciendo uso de las nociones de 'función manifiesta' y 'función latente', la sociedad por la práctica creativa de sus actores.

\section{Presentación informal de la TGR}

Así pues, la TGR pretende explicar la conducta del individuo en lo que concierne a su actitud de asimilar/adaptarse-a los valores de un colectivo, en el sentido de vincularla a su aspiración de afiliarse-a/integrarse-en ${ }^{3}$ este colectivo.

El concepto de grupo de referencia se originó en la psicología social; sin embargo, Merton defiende que "el concepto de grupo de referencia tiene también un lugar distintivo en la teoría de la sociología, con su enfoque

3 Tanto para el caso de "asimilar/adaptarse-a" como para el de "afiliarse-a"/integrarse-en" los pares de expresiones pueden considerarse sinónimas, siendo más apropiado un miembro del par que otro según el contexto de uso. A fin de evitar lo farragoso que sería mantener los pares de expresiones a lo largo del texto, a partir de aquí privilegiaré el uso de "asimilar" y de "afiliarse a" (prescindiendo en este caso del guión del que hice uso previamente). 
sobre la estructura y funciones de los ambientes sociales en que están situados los individuos" (Merton, 1968, p. 335 [2002, p. 362]). En todo caso, es importante aclarar que "la expresión ahora consagrada de 'grupo de referencia' tiene algo de inapropiado, porque se aplica no sólo a grupos, sino que también a individuos y a categorías sociales" (Merton, 1968, p. 338 [2002, p. 365]).

Un individuo de referencia es aquél que tiene cierto status social diferenciado, como puede ser un artista renombrado, un importante deportista, etc. Reconociendo que, por una cuestión de simplicidad, en la tradición sociológica se utiliza en general la expresión 'grupo de referencia' para designar también individuos de referencia, Merton procura aclarar esta última noción, y lo hace caracterizándola a partir de sus semejanzas y diferencias con respecto a la noción de papeles sociales.

El individuo de referencia ha sido descrito con frecuencia como un modelo de papel. Pero, como implican las palabras mismas, el supuesto de que sean cosas conceptualmente sinónimas oscurece una diferencia fundamental en las materias de las cuales se refieren respectivamente. La persona que se identifica con un individuo de referencia tratará de aproximarse a la conducta y valores de aquel individuo en sus diferentes papeles sociales. El concepto de modelo de papel puede tomarse en un sentido más restringido que denota una identificación más limitada con un individuo sólo en uno o en unos pocos papeles seleccionados. (Merton, 1968, pp. 656-357 [2002, p. 384])

En lo que se refiere a los grupos "se entiende en general que el concepto sociológico de grupo se refiere a un número de personas que actúan entre sí de acuerdo con normas establecidas" (Merton, 1968, p. 339 [2002, p. 366]). Las relaciones sociales consagradas y características (formas normadas de interacción) de cada grupo acaban por caracterizarse como aspectos identificables de la estructura social.

A su vez, las categorías sociales son caracterizadas por Merton como "agregado de situaciones sociales cuyos ocupantes no están en interacción social. Tienen características sociales idénticas — de sexo, edad, situación matrimonial, ingreso, etc.- , pero no están orientadas necesariamente hacia un cuerpo de normas distintivo y común" (Merton, 168, p. 353 [2002, p. 381]).

Es importante en este contexto tener presente que tiene que establecerse una relación entre un individuo y un grupo (que puede estar dado por un único individuo, como ya se señaló) para que éste pueda servir como referencia de aquél. Esto queda más claro si percibimos "que el concepto de la conducta relativa a grupo de referencia presupone algún conocimiento o imagen de las normas y los valores predominantes en el grupo" (Merton 1968, p. 391 [2002, p. 419]). Para concretar puede decirse que la TGR 
"se enfoca sobre el proceso mediante el cual los individuos se relacionan con los grupos y refieren su conducta a los valores de los grupos" (Merton, 1968, p. 280 [2002, p. 306]). Por 'valores del grupo' se debe entender cosas como 'normas', 'cánones', 'patrones', 'hábitos', 'prácticas', 'rutinas', 'usos', 'modas', 'costumbres', 'tradiciones', 'etiqueta', 'protocolos', 'ritos', 'procedimientos', 'reglas', etc. La referencia a estos valores puede ser: (i) positiva, cuando "comprende la asimilación motivada de las normas del grupo o de los patrones del grupo" (Merton, 1968, p. 354 [2002, p. 382]); o (ii) negativa, cuando hay "el rechazo motivado, es decir, no sólo la mera no aceptación de las normas, sino que la formación de contranormas" (Merton, 1968, p. 354 [2002, p. 382]); o (iii) neutra, cuando los individuos son completamente indiferentes a la perspectiva de pertenecer o no pertenecer al grupo - esta clase de individuos consta de los que no se orientan en absoluto hacia el grupo ni positiva ni negativamente, pero que podrían hacerlo pues tienen cierta interacción con (conocen) el grupo en cuestión ${ }^{4}$.

Los comentarios previos parecen dar a entender que los grupos de referencias son sin más determinados colectivos o grupos de individuos; es decir, lo que podríamos representar como ciertos conjuntos de individuos (incluidos algunos conjuntos unitarios). Sin embargo, considerarlos así conlleva perder un rasgo decisivo de los grupos de referencia, a saber, que un determinado colectivo o grupo de individuos pasa a ser grupo de referencia en tanto que lo es para un determinado individuo en determinada circunstancia. No hay grupos de referencia en términos absolutos, los hay relativamente a un individuo y una circunstancia. Que ser grupo de referencia es relativo a un individuo debe resultar intuitivamente obvio, toda vez que ser grupo de referencia parece que requiere la adopción de una actitud, un posicionamiento determinado, por parte de ese individuo. Que sea relativo a una circunstancia parece que es apropiado por un par de razones. En primer lugar, lo que sea un grupo de referencia para un individuo en un determinado momento no tiene por qué serlo en otro momento. Esta mera razón

${ }^{4}$ La noción de referencia neutra sirve para capturar un fenómeno que es distinto, por ejemplo, del caso en el que el individuo no pertenece a un grupo y es indiferente a pertenecer o no a él porque no está en la órbita de este grupo; o sea, ni siquiera mantiene algún tipo de relación con él. La noción de referencia neutral captura el fenómeno donde un individuo mantiene relación con un grupo (sea como sea esta relación) y, sin embargo, este grupo no sirve (no está como) guía para este individuo; o sea, no se da el caso de ser ni referencia positiva ni referencia negativa para este individuo. Estrictamente no se da la referencia, pero eso no le quita (a la noción de referencia neutral) su valor (y utilidad). Es común la utilización de una noción similar en las encuestas (sea para publicidad, actividades políticas u otras) donde se procura identificar, por ejemplo, la aceptación y rechazo de cierto producto o de cierta persona por parte de la población. 
daría lugar a que en vez de "circunstancias" se pensara en que la noción de "grupo de referencia" estuviera relativizada a "momentos" (además de estarlo a "individuos"), pero lo cierto es que en el mismo momento un mismo individuo podría considerar dos o más colectivos como grupos de referencia para él (por ejemplo, para un chico dado, una pandilla del barrio, por un lado, y un grupo de compañeros en el centro de estudios, por otro lado). Por eso, en segundo lugar, debe considerarse una noción que intuitivamente sea más compleja que la de "momentos temporales" (que capture la idea de contexto estructural), llamémosla "circunstancia", respecto a las cuales se fija qué colectivos son grupos de referencia; aunque a los efectos de este trabajo las presentaremos como entidades simples. Preservaremos, pues, la noción de grupo de referencia como una noción dependiente de otras tres más básicas; a saber: "individuos", "colectivos" 5 y "circunstancias".

Teniendo esto en cuenta, podemos decir que la hipótesis central de la teoría es que

en la medida en que los individuos de un grupo subalterno o en perspectiva se sienten movidos a afiliarse a un grupo, tenderán a asimilarse a los sentimientos y adaptarse a los valores del estrato del grupo que tiene autoridad y prestigio. (Merton, 1968, p. 308 [2002, pp. 334-335])

En base a esto, Merton afirma que en casos como los de

[u]n soldado raso del ejército que busca el ascenso, sólo en un sentido estrecho y teóricamente superficial puede considerarse que siga una conducta diferente de la de un inmigrante que se asimila los valores de un grupo nativo, o de un individuo de clase baja que se ajusta a su concepto de las normas de conducta de la clase media alta, o de un muchacho en una zona de barrios bajos que se orienta hacia los valores de un trabajador de casa de beneficencia y no hacia los valores de la pandilla de la esquina, o de un estudiante de Bennington que abandona las ideas conservadoras de sus padres para adoptar las ideas más liberales de sus compañeros de colegio universitario, o de un católico de clase humilde que se aparta de la norma de su intra-grupo votando por los republicanos, o de un aristócrata francés del siglo XVIII que se alinea en un grupo revolucionario de la época. (Merton, 1968, p. 332 [2002. pp. 359-360])

Todos ellos son casos que responden a la pauta dada por la TGR.

Parece claro que la conducta de un individuo, según nos propone Merton, se caracteriza por ser la expresión de un sentimiento por parte de ese individuo en relación al grupo. Según sea este sentimiento, el grupo será de

${ }^{5}$ Hasta aquí hablé de "colectivos" o "grupos". A partir de ahora privilegiaré la expresión de "colectivos" para evitar confusiones entre las expresiones de "grupo" y de "grupo de referencia". Además, como se verá, entre los colectivos se incluyen colectivos unitarios (es decir, de un solo individuo), a fin de poder disponer de éstos como valores para la noción de "grupo de referencia". 
referencia positiva, negativa o neutra. Puede ser el caso de una "asimilación motivada de las normas del grupo", "[d]el rechazo motivado" de dichas normas (Merton, 1968, p. 354 [2002. p. 382]) o de la neutralidad; es decir, referencia positiva, negativa y neutra, respectivamente. En este sentido, aspirar a pertenecer, por ejemplo, supone aceptación de los valores del grupo. Querer "ser del grupo", "ser como los del grupo", es (presupone) aceptar lo que acepta el grupo. Aspirar a abandonar al grupo es (supone) rechazar lo que acepta el grupo. Y finalmente, están aquellos que no se orientan en absoluto hacia el grupo en cuestión.

\section{Los modelos potenciales de la TGR}

Para la Metateoría Estructuralista las teorías son entidades modelo-teóricas y, por tanto, presentar una teoría requiere, entre otras cosas, presentar una clase de modelos ${ }^{6}$. El primer paso para ese cometido es dar cuenta de los sistemas de los que cabe preguntarse si son o no son modelos de una teoría, es decir, de lo que en la corriente estructuralista se conoce como los modelos potenciales de dicha teoría. En este apartado se presentan los modelos potenciales de la TGR. Pero antes veamos una aproximación informal de lo que se entiende por modelos potenciales de una teoría dada $T$. $\mathrm{Al}$ respecto dice Moulines:

El conjunto de modelos potenciales (al cual, siguiendo la nomenclatura estándar de Stegmüller, designaremos por $M_{p}$ ) viene determinado por las condiciones estructurales o "axiomas impropios" que intervienen en la caracterización de un predicado conjuntista correspondiente a la axiomatización de la teoría en cuestión. Intuitivamente, un modelo potencial de una teoría dada $T$ es cualquier sistema del que sabemos que tiene la estructura conceptual requerida para ser un modelo de $T$, aunque posiblemente no sepamos si cumple realmente las leyes empíricas (los "verdaderos axiomas") de $T$ y por lo tanto no sepamos si efectivamente es un modelo de $T$. Con las estructuras contenidas en $M_{p}$ "conceptuamos la realidad" de determinada manera (de la manera correspondiente al lenguaje de $T$ ). Pero con ello, naturalmente, aún

${ }^{6}$ Además de entender que presentar una teoría requiere, entre otras cosas, presentar una clase de modelos, la Metateoría Estructuralista se distingue de las demás concepciones semánticas por refinar la noción de teoría. Para la concepción estructuralista, el tipo más simple de estructura conjuntista que pudiera ser identificado con, o pudiera servir como reconstrucción lógica de, una teoría empírica es denominado elemento teórico y puede ser representado, en una primera aproximación, con el par ordenado consistente en el núcleo $K$ y el campo de aplicaciones intencionales $I: T=\langle K, I\rangle$. El núcleo $K$, que constituye la identidad formal de una teoría, es un quíntuplo ordenado $\left\langle M_{p}, M, M_{p p}, C, L\right\rangle$. En la medida que, estas nociones aparezcan en el trabajo serán presentadas con más detalle. 
no queda garantizado que esa conceptuación sea empíricamente válida y fructífera. (Moulines, 1986. p. 300)

En el caso de la TGR entre los constituyentes de la teoría tenemos: "individuos", "colectivos", "valores" y "circunstancias". A ellos hay que añadir un dominio auxiliar que denominamos "evaluaciones". Éstos son los dominios a partir de los cuales se darán (conjuntistamente) las funciones "normativizar", “comportarse" y "aspirar", además de la relación "grupo de referencia". Teniendo en cuenta estos componentes conceptuales (y presuponiendo que el campo de acción del individuo está limitado por el conjunto de relaciones que tiene con los distintos colectivos existentes), ya es posible caracterizar el conjunto de los modelos potenciales de la TGR.

D1:

$\mathbf{M}_{\mathrm{p}}(\mathrm{TGR}): x$ es un modelo potencial de la Teoría de los Grupos de Referencia $\left(x \in \mathrm{M}_{\mathrm{p}}(\mathrm{TGR})\right)$ syss existen $I N D, C O L, V A L, C I R, E V A$, nor, com, asp, $G R U$, tales que:

(1) $x=\langle I N D, C O L, V A L, C I R, E V A$, nor, com, asp, GRU $\rangle$

(2) IND es un conjunto finito, no vacío ("individuos")

(3) $C O L \subseteq(\operatorname{Pot}(I N D)-\varnothing)$ ("colectivos")

(4) VAL es un conjunto finito, no vacío ("valores")

(5) CIR es un conjunto finito, no vacío ("circunstancias")

(6) $E V A=\{+,-$, ? $\}$ ("evaluaciones"), es un dominio auxiliar

(7) nor: $C O L \times C I R \rightarrow(\operatorname{Pot}(V A L)-\varnothing)$ ("normativizar (o asignar valores)")

(8) com: IND $\times$ CIR $\times(\operatorname{Pot}(V A L)-\varnothing) \rightarrow E V A$ ("comportarse")

(9) asp: IND $\times C I R \times C O L \rightarrow E V A$ ("aspirar")

(10) GRU $\subseteq C O L \times I N D \times C I R$ ("grupo de referencia, para un individuo en una circunstancia")

Comentarios a este predicado:

(1) Presenta la estructura de los modelos potenciales, conformada según los componentes conceptuales característicos de la TGR.

(2) IND representa un conjunto de seres humanos (o individuos). Personas que llevan su vida relacionándose con otras, siendo parte de determinados contextos sociales y no siendo parte de otros, inmersas en la cultura de su lugar (su lugar puede ser desde un pueblito de pescadores hasta el planeta como un todo, dependiendo entonces de las actividades y el nivel cultural-económico de cada individuo).

(3) COL es un conjunto de colectivos de individuos. Desde un "colectivo unitario" formado por un solo individuo, pasando por grupos de personas con cierta identidad de grupo asumida por sus miembros 
(identidad que proporcionan unas normas, más o menos explícitas, compartidas), hasta, incluso, categorías sociales determinadas por una característica que comprenden fenómenos como, por ejemplo, los "individuos de un sexo" o las "clases sociales". Lo que es distintivo de estos colectivos, así caracterizados, es que tienen como condición necesaria el estar constituidos por individuos. Pueden ser grupos que existen actualmente o que no existan más (es decir, que hayan existido y hayan terminado desapareciendo), pueden ser grupos cerrados (aquellos que no permiten o dificultan en mucho el ingreso de nuevos integrantes) o abiertos (aquellos que permiten el ingreso de nuevos integrantes, muchos incluso los buscan constantemente), pueden ser grupos a los cuales se pertenece o no, pueden ser grupos a los cuales ya se perteneció y hoy ya no se pertenece; un colectivo puede ser un grupo al que se aspire ingresar, del que resulte indiferente la afiliación, o incluso del que se tenga razones para desear no pertenecer. No toda posible combinación de individuos es uno de estos colectivos, sólo ciertas combinaciones lo son (por ejemplo, no sería un colectivo para nuestra teoría el resultante de considerar conjuntamente un individuo de Caracas, otro de Londres y otro de Praga que no tuvieran nada socialmente relevante que ver entre sí).

(4) VAL es el conjunto de valores: normas, cánones, patrones, hábitos, rutinas, usos, modas, costumbres, tradiciones, etiquetas, protocolos, ritos, procedimientos, prácticas, reglas..., que están presentes en la sociedad constituyendo la moral de los grupos sociales. Los valores son principios que permiten orientar los comportamientos (o conductas) a adoptar; son creencias fundamentales que ayudan a preferir, evaluar y elegir unas cosas en lugar de otras, o unos comportamientos (o conductas) en lugar de otro; son normas que rigen las conductas.

(5) CIR es el conjunto de contextos estructurales o circunstancias. Captura el estado de cosas que sirve de escenario para que puedan “actuar" los individuos y los colectivos. Aunque podría ser caracterizada como una noción compleja (con estructura), no se hace así en esta reconstrucción por entenderse que la matización en nada contribuiría para un mejor entendimiento de la teoría en tanto que autónoma, tal y como se la considera aquí; es decir, siendo bastante clara la noción intuitiva de circunstancia, presentarla como una estructura no afectaría en nada lo que es central e importante para la reconstrucción a la luz de la consideración autónoma que de la 
misma aquí se hace (otra cosa es que pueda tener interés esa consideración más estructurada de la circunstancias en un marco más amplio de teorías del que forme parte la TGR).

(6) EVA es un conjunto de elementos auxiliares. Sus elementos son los tres tipos de posibilidades que cabe considerar al hacer evaluaciones, es decir, sus elementos son la posibilidad positiva (+), la negativa (-) y la neutra (?). El uso de estos elementos en la reconstrucción es algo peculiar y, justamente por eso, será aclarado más adelante cuando aparezcan en las funciones correspondientes. En todo caso, como se verá de inmediato, son los valores que le corresponden al codominio de las funciones com y asp.

(7) nor es la función que a un colectivo le asigna, en determinada circunstancia, ciertos valores. Esto es importante para la TGR una vez que estos valores estarán (o no) directamente involucrados con el comportamiento del individuo. Es en base a estos valores que se tiene la función "comportarse".

(8) com es la función que a un individuo, en determinada circunstancia, atribuye una relación particular respecto a un conjunto de valores, puede ser de tres maneras: i) de asimilar este conjunto de valores $(+)$, ii) de rechazar a este conjunto de valores (-) o iii) de ser indiferente con respecto a este conjunto de valores (?).

(9) asp es la función que a un individuo, en una circunstancia, atribuye una relación particular respecto a un colectivo, puede ser de tres maneras: i) de aspirar a pertenecer a este colectivo (+), ii) de aspirar a no-pertenecer a este colectivo (-) o iii) ser indiferente con respecto a pertenecer a este colectivo (?). Captura la actitud del individuo hacia el colectivo.

(10) GRU es una relación entre colectivos, individuos y circunstancias, de manera que determinado colectivo es grupo de referencia para un individuo en cierta circunstancia. Así pues, vincula el comportamiento (conducta) del individuo, por medio de los valores, a un colectivo. Este vínculo es lo que hace que el colectivo pase a ser grupo de referencia para este individuo en lo que se refiere a este comportamiento (conducta). Cuando i) el individuo asimila el conjunto de valores de colectivo, hay referencia positiva, ii) el individuo rechaza el conjunto de valores del colectivo, hay referencia negativa iii) el individuo es indiferente, bajo las condiciones ya mencionadas, con respecto al conjunto de valores del colectivo, hay referencia neutra. 


\section{Los modelos actuales de la TGR}

Los modelos de una teoría son las entidades que satisfacen la totalidad de las condiciones introducidas, es decir, presentan las restricciones que introduce la teoría a este último. En el caso de la TGR, es posible expresarlos del siguiente modo:

D2:

M(TGR): $x$ es un modelo potencial de la Teoría de los Grupos de Referencia ( $x \in \mathrm{M}(\mathrm{TGR})$ ) syss existen IND, COL, VAL, CIR, EVA, nor, com, asp, GRU, tales que:

(1) $x=\langle I N D, C O L, V A L, C I R, E V A$, nor, com, asp, GRU $\rangle$

(2) $x \in \mathrm{M}_{\mathrm{p}}$ (TGR)

(3) para todo ind $\in I N D$, col $\in C O L, v_{\mathrm{i}(i \in \mathbb{N}, 1 \text { sisn })} \in V A L$, cir $\in C I R, \alpha \in E V A$, y siendo $\left\{v_{1}, \ldots, v_{\mathrm{k}}\right\} \in(\operatorname{Pot}(V A L)-\varnothing)$ :

$G R U(c o l$, ind, cir $) \leftrightarrow$ (si se diera que asp(ind, cir, col) $=\alpha$ y que nor $($ col, cir $)=\left\{v_{\mathrm{g}}, \ldots, v_{\mathrm{k}}\right\}$, entonces se daría que com (ind, cir, $\left\{v_{\mathrm{g}}, \ldots\right.$, $\left.\left.\left.v_{\mathrm{k}}\right\}\right)=\alpha\right)$

Comentario sobre este predicado

(1) Presenta la estructura de los modelos potenciales, conformada según los componentes conceptuales característicos de la TGR.

(2) Expresa la condición formal de que $x$, para ser modelo de la TGR, pertenezca a los modelos potenciales de la TGR.

(3) Un colectivo es un grupo de referencia para un individuo en determinada circunstancia syss (si se diera que la aspiración del individuo en esa circunstancia a afiliarse al colectivo tiene la evaluación $a$ y la normativa del colectivo en cuestión en esa circunstancia viene dada por los valores $\left\{v_{1}, \ldots, v_{\mathrm{k}}\right\}$, entonces se daría que el comportamiento del individuo en esa circuntancia respecto a esos valores - normativa- tiene la evaluación $\alpha$ ). Nótese que $\alpha$ debe tener uno de los tres valores que pertenecen a $E V A$, y que tras el bicondicional se requiere que si el valor de asp es $\alpha$, el valor de com sea el mismo.

\section{Los modelos potenciales parciales de la teoría}

Es importante tener presente que

Los modelos parciales de una teoría $T$ son las estructuras que describen, mediante conceptos no-teóricos o "empíricos" relativamente a $T$, los sistema posibles a los que es concebible aplicar $T$. Constituyen, por así decir, la "base empírica” de $T$ (en el sentido relativo). Su construcción no presupone ni los conceptos específicos de $T$ 
ni sus principios. Generalmente son otras teorías, previas a $T$, las que proveen los modelos parciales de $T$. Al conjunto de todos los modelos parciales de una teoría dada lo llamamos $M_{p p}$. Este conjunto queda determinado por un predicado conjuntista definidos por los mismos axiomas estructurales que determinan $M_{p}$ exceptuando aquellos que se refieren a los conceptos T-teóricos. (Moulines, 1986. p. 300)

Teniendo esto en cuenta, se puede examinar los conceptos básicos de TGR, a saber, IND, COL, VAL, CIR, EVA, nor, com, asp, GRU, a fin de determinar cuáles de estos conceptos son TGR-teóricos y cuáles son TGRno-teóricos, con la mente puesta en que los TGR-teóricos presuponen el principio o ley de la TGR para la determinación de sus valores.

Comencemos con IND. Considérese la caracterización informal que dimos de este concepto al explicitar los modelos potenciales de TGR. Así pues, IND constituye uno de los conjuntos básicos principales que establecen la ontología empírica de la teoría. Sin embargo, para averiguar si una entidad particular pertenece al conjunto IND no es necesario presuponer la validez de la ley de TGR; bastarían, de hecho, métodos empíricos independientes, aquellos que utilizamos para identificar observacionalmente los seres humanos y distinguirlos de las demás cosas existentes en nuestra vida cotidiana.

Algo similar ocurre con COL. Este concepto es también un concepto que constituye otro de los conjuntos principales que establecen la ontología empírica de la teoría; sin embargo, se trata de un conjunto derivado de IND. A pesar de ese carácter derivado y dado su papel especialmente relevante en la teoría, voy a hablar de la entidad que designa como "conjunto (o dominio) básico" forzando dicha noción. Que un conjunto de individuos cualquiera pertenezca a $C O L$ se determina empíricamente, con independencia de la ley fundamental de la TGR. La observación del comportamiento y/o de algunas de las características de los individuos nos permite determinar las entidades que pertenezcan a dicho conjunto. Con cada sujeto humano podemos conformar un colectivo, dado extensionalmente por el conjunto unitario al que ese sujeto pertenece. Un grupo es identificado como tal por una semejanza en las actitudes, las acciones, los comportamientos de sus integrantes. En algunos casos semejanzas geográficas, educacionales, tecnológicas - pero todas ellas con la característica de que hay interacción entre los individuos- La determinación de sus integrantes puede darse por una sencilla observación (en muchos y variados casos) o por una investigación histórica por relatos orales o bibliográficos que relaten las actitudes, las acciones, los comportamientos de sus integrantes (grupos que no existen más). En grupos formales, como asociaciones, es posible buscar en los registros de la asociación el listado de integrantes, o sea, los documentos de 
la asociación, la carpeta para tomar lista en una clase, el anuncio con los listados de profesores del departamento de determinada disciplina y muchos otros documentos posibilitan la determinación. Por fin, en cuanto a las categorías sociales, la semejanza puede ser económica, educacional, tecnológica, etc., pero no necesariamente con interacción entre los individuos. La determinación de las entidades que componen este tipo de colectivo, una vez que se tenga establecido el criterio (por ejemplo, varones entre 50 y 70 años de determinada ciudad), se establece por medio de encuestas, de acceso a bancos de datos que contengan dicha información.

También VAL constituye otro de los conjuntos básicos principales que establecen la ontología empírica de la teoría, comprende los valores determinados empíricamente, con independencia de la TGR. La determinación de las entidades que caen bajo este concepto puede darse por el acceso a estatutos, códigos de leyes (normas y reglas), por publicaciones especializadas (moda), por manuales (etiqueta, protocolo, rito, procedimiento), etc.

En lo que se refiere a CIR, el conjunto de contextos estructurales o circunstancias, parece darse lo mismo. Considerando la caracterización informal que dimos de este concepto al explicitar los modelos potenciales de la TGR, debe recordarse que ésta es una noción intuitiva idealizada. De hecho es una noción vaga que podría comprender, además del componente temporal, otros factores respecto a los que relativizar, por ejemplo, la combinación de valores asumida por un colectivo o el comportamiento de un colectivo. Entre esos factores encontraríamos eventos (que incluyen el componente temporal) o aspectos geográficos junto a un componente temporal (considérese cómo los valores asumidos por un grupo pueden, en determinado momento, estar condicionados por eventos como un estallido social o por estar viviendo en una determinada zona). La determinación de las circunstancias para cada aplicación de la TGR es establecida por los científicos (sociólogos) por convención, a la luz del problema (aplicación pretendida) a considerar. La convención no tiene por qué ser arbitraria, puede darse razón de la misma en función del problema considerado.

Consideramos EVA como un conjunto auxiliar, por lo que estrictamente no formaría parte de los conceptos descriptivos característicos de la TGR. Sus entidades podrían ser, convencionalmente, tres cualesquiera, que representen la evaluación positiva, la negativa y la neutra. Aquí hemos elegido “+”, “-” y “?”. Sin embargo, parece conveniente que figure explícitamente en la estructura, aun tratándose de un conjunto auxiliar, ya que proporciona valores del codominio de dos funciones. Considerar los conjuntos auxiliares explícitamente en la estructura es un procedimiento habitual cuando 
parece conveniente para una mayor claridad conceptual de la teoría a identificar.

Claramente, todos los dominios básicos empíricos de la teoría son TGRno teóricos. Todos ellos pueden ser determinados de forma independiente de la teoría. Téngase presente que EVA, en tanto que dominio auxiliar, no es ni TGR-teórico ni TGR-no-teórico, ya que no da cuenta de un dominio empírico, es decir, no corresponde a un concepto empírico. Veremos ahora lo que pasa en el caso de las funciones y la relación que presenta la TGR.

En lo que respeta a nor, es la función que a un colectivo le asigna, en determinada circunstancia, cierta combinación de valores. Claramente esta función es determinada de modo independiente de la TGR. La determinación de la combinación de valores que caracteriza un colectivo en determinada circunstancia puede darse a partir del comportamiento de los integrantes de los colectivos, cuando estos comportamientos manifiestan dichos valores. También puede darse por medio de la expresión del individuo (cuando conforma un colectivo unitario) o, por ejemplo, de líderes (para grupos). Puede determinarse el valor de la función por una investigación en documentos oficiales tales como los estatutos (grupos formales). Asimismo usando procedimientos técnicos más complejos. Por ejemplo, los valores asignados en determinadas circunstancias a las categorías sociales pueden ser determinados por encuestas, como, por ejemplo, aquellas en las cuales los encuestados tienen que contestar ciertas cuestiones para saber si cada uno está habilitado a continuar haciendo la encuesta o no, y con ello determinar si forma parte o no de cierto colectivo; en caso de estar habilitado (esté dentro de los parámetros establecidos para la encuesta) se le pregunta acerca de lo que le resulta más importante, lo que le gusta, lo que no le gusta, y con ello se establecen la combinación de valores para ese individuo. La comparación del resultado de estas encuestas determina los valores de determinada categoría social.

También com, la función que asigna a un individuo, en determinada circunstancia, la aceptación activa, el rechazo activo o la indiferencia dado la actitud del individuo con respecto a un conjunto (una combinación) de valores. Los valores que adquiera la función pueden ser determinados de modo independiente de la teoría una vez que se tengan establecidos los argumentos de la función. Dado un individuo y la circunstancia relevante, una vez determinada una combinación de valores, la observación de la actividad del individuo nos permite identificar cuál de las tres posibilidades $(+,-$, ?) es el caso.

En cuanto a asp, la función que a un individuo, en una circunstancia, atribuye una relación particular respecto a un colectivo, se recuerda que 
dicha relación puede ser de tres maneras: i) de aspirar a pertenecer a este colectivo (+), ii) de aspirar a no-pertenecer a este colectivo (-) o iii) ser indiferente con respecto a pertenecer a este colectivo (?). La función captura la actitud del individuo hacia el colectivo. Ésta también es una función que puede tener su valor determinado de modo independiente de la TGR. Un método posible para dicha determinación es preguntarle al individuo directamente acerca de determinado colectivo si él aspira pertenecer, si aspira no pertenecer o si es indiferente cuanto a eso. Otro modo de determinación sería la utilización de técnicas como la aplicación de cuestionarios o la realización de dinámicas de grupo. En ninguno de los tres casos es necesario presuponer la ley fundamental de la TGR para que sea posible la determinación.

Finalmente tenemos GRU, una relación entre colectivos, individuos y circunstancias. Para que se pueda determinar qué colectivo satisface la relación $G R U$, dado un individuo determinado y una circunstancia determina$\mathrm{da}$, se requiere que se cumplan ciertas condiciones se obtenga un resultado; las condiciones son: 1) que la aspiración del individuo en esa circunstancia a afiliarse a un colectivo tenga una evaluación $a$, es decir, que sea determinable la aspiración del individuo considerado, en cierta circunstancia, respecto a un determinado colectivo; y 2) que sea determinable la normativa del colectivo en cuestión, en esa circunstancia, como una determinada combinación de valores $\left\{v_{1}, \ldots, v_{\mathrm{k}}\right\}$. El resultado debe ser que el comportamiento del individuo tenga la evaluación $a$, es decir, el mismo valor que el obtenido para asp al aplicar esa función al individuo en cuestión, en la circunstancia considerada, respecto al mismo colectivo. Dicha relación entre condiciones y resultado forma parte de lo que es la ley fundamental de la TGR: la relación entre las condiciones 1) y 2), por un lado, y el resultado implicado (por medio de un condicional subjuntivo), por otro lado, constituyen el consecuente del bicondicional que expresa la ley fundamental de la TGR. Única y exclusivamente de este modo es posible determinar $G R U^{7}$. No hay otro condicional subjuntivo que permita establecer si un colectivo es un grupo de referencia para un individuo en cierta circunstancia: no hay otra condición de prueba respecto a la que obtener un determinado resultado que permita establecer que un colectivo en determinada circunstancia es un grupo de referencia para un individuo. De este modo GRU se caracteriza como una relación TGR-teórica, pues su determinación es dependiente de la TGR.

${ }^{7} \mathrm{El}$ concepto de grupo de referencia se caracteriza como un concepto relacional disposicional. Más adelante trataré de modo más detallado de esta peculiaridad del concepto. 
Sabiendo que apenas $G R U$ es constituyente del conjunto de términos TGR-teóricos ya se puede caracterizar a los modelos potenciales parciales de la teoría.

D3:

$\mathbf{M}_{\mathrm{pp}}$ (TGR): $y$ es un modelo potencial parcial de la Teoría de los Grupos de Referencia $\left(y \in \mathrm{M}_{\mathrm{pp}}(\mathrm{TGR})\right)$ syss existen IND, COL, VAL, CIR, EVA, nor, com, asp, GRU tales que:

(1) $x=\langle I N D, C O L, V A L, C I R, E V A$, nor, com, asp, GRU $\rangle$

(2) $x \in \mathrm{M}_{\mathrm{p}}$ (TGR)

(3) $y=\langle I N D, C O L, V A L, C I R, E V A$, nor, com, asp $\rangle$

\section{Las aplicaciones intencionales de la TGR}

El dominio de aplicaciones intencionales de TGR constituye la clase de aquellos sistemas empíricos a los que se desea aplicar la ley fundamental de dicha teoría. Estos sistemas no pueden ser caracterizados por medios puramente formales y además, lo único que se puede decir desde el punto de vista formal es que $\mathrm{I}(\mathrm{TGR}) \subseteq \mathrm{M}_{\mathrm{pp}}$ (TGR). Entre los posibles sistemas empíricos que satisfacen dicha condición formal, los que pertenecen al conjunto de las aplicaciones intencionales de una teoría se decide pragmáticamente por parte de la comunidad de científicos. Algunos ejemplos de estos sistemas, como visto, según Merton (1968, p. 323 [2002, p. 350]), son:

a) El hecho de que sólo en un sentido estrecho y teóricamente superficial puede considerarse que el soldado raso del ejército que busca el ascenso siga una conducta diferente de la de un inmigrante que se asimila los valores de un grupo nativo. En realidad son dos aplicaciones comparadas por su similitud estructural: por un lado, un individuo, en la circunstancia de ser soldado raso del ejército y buscar ascenso [asp $(+)]$, según la propuesta de analogía entre las aplicaciones, asimila $[\operatorname{com}(+)]$ los valores de [nor] un colectivo superior en la jerarquía; por otro lado, un individuo, en la circunstancia de ser un inmigrante y asimilar $[\operatorname{com}(+)]$ los valores de [nor] un colectivo nativo, otra vez, según la propuesta de analogía, quiere [asp (+)] integrarse a este colectivo. En este ejemplo existen dos individuos distintos que presentan el mismo comportamiento referente a los valores de sus respectivos colectivos. La analogía entre las dos aplicaciones puede considerarse el tipo de procedimiento descrito por Kuhn (1962) para expandir las aplicaciones de una teoría a partir de algunas paradigmáticas aprendiendo a ver cuáles son los aspectos relevantes de la semejanza entre las aplicaciones paradigmáticas al ampliar las aplicaciones pretendidas a nuevos casos. 
b) El hecho de que un muchacho en una zona de barrios bajos se oriente hacia los valores de un trabajador de casa de beneficencia y no hacia los valores de la pandilla de la esquina. En este caso se da una situación en la que se pueden observar dos comportamientos distintos: por un lado i), un individuo, en la circunstancia de ser un joven de una zona de barrios bajos y no orientarse a la pandilla de la esquina, se oriente hacia $[\operatorname{com}(+)]$ los valores de [nor] un trabajador de casa de beneficencia (colectivo) y, por otro lado ii), este mismo individuo en la circunstancia de ser un joven de una zona de barrios bajos y orientarse hacia los valores de un trabajador de casa de beneficencia, no se oriente hacia [com (?)] los valores de [nor] la pandilla de la esquina (colectivo). Importa recordar que no orientarse a tales valores no es el mismo que orientarse negativamente a dichos valores. Mismo con la vaguedad del ejemplo propiciado por Merton, parece ser el caso que el individuo desea [asp] participar del colectivo de los trabajadores de casas de beneficencia y es indiferente [asp] respeto a la pandilla de la esquina. El comportamiento con referencia a los valores de un colectivo es distinto del comportamiento con referencia a los valores de otro. Cada caso, estrictamente, es una aplicación diferente de la TGR, aunque con una interdependencia entre las mismas, interdependencia de la que no se extraen consecuencias relevantes por la TGR.

c) El hecho de que un estudiante de Bennington abandone las ideas conservadoras de sus padres para adoptar las ideas más liberales de sus compañeros de colegio universitario. También en este caso se da una situación de la cual se puede observar dos comportamientos distintos: por un lado i), que un individuo en la circunstancia de ser estudiante de Bennington y de no adoptar hasta entonces ideas liberales, abandona $[\mathrm{com}(-)]$ las ideas conservadoras (valores) de [nor] sus padres (colectivo) y, por otro lado ii), este mismo individuo, en la circunstancia de ser estudiante de Bennington y ter adoptado posiciones conservadoras en el pasado, adopta $[\mathrm{com}(+)]$ las ideas más liberales (valores) de $[$ nor] sus compañeros de colegio universitario (colectivo). El comportamiento con referencia a los valores de un colectivo es distinto del comportamiento con referencia a los valores de otro. Nuevamente, cada caso, estrictamente, es una aplicación diferente de la TGR, aunque con una interdependencia entre las mismas, interdependencia de la que no se extraen consecuencias relevantes por la TGR.

El hecho de que dos (los ejemplos b) y c)) de los tres ejemplos traigan una situación en la cual se puede observar dos comportamientos pudiera llevar a pensar que la aplicación de la TGR requiere la comparación entre dos comportamientos. Aunque sea más intuitiva la presentación de los ejemplos de este modo, no cabe de hecho contemplar este tipo de comparación 
para la aplicación de la teoría; los dos comportamientos se conciben como aplicaciones autónomas de la TGR. El ejemplo a) muestra esto claramente. Lo más que cabe es, dado un comportamiento $x$ cualquiera que pudiera ser comparado con el propiamente considerado en la aplicación, es integrar $x$ como parte de la circunstancia de dicha aplicación.

\section{Observaciones acerca del carácter relacional y disposicional de la noción teórica GRU}

La reconstrucción de la TGR mostró que el concepto "grupo de referencia" es un concepto relacional disposicional, o sea, el concepto captura una relación que para efectuarse requiere que si se dieran ciertas condiciones se obtendría determinado resultado. En realidad, tal y como se indicó, "grupo de referencia" abrevia "grupo de referencia para un individuo en cierta circunstancia".

En lo que respecta al carácter relacional del concepto, como se habrá observado, un determinado colectivo es un grupo de referencia relativamente a un individuo y una circunstancia. De esta forma qué colectivo sea grupo de referencia depende del individuo que se considere, pero de la circunstancia también; si cambia la circunstancia, y aunque el individuo sea el mismo, puede darse que otro colectivo sea grupo de referencia para él, o que no lo sea ninguno. Para que un colectivo sea grupo de referencia para un individuo en una circunstancia, es necesario y suficiente, como ya se señaló, que se cumpla el condicional subjuntivo que forma parte de la ley de TGR.

En lo que respeta al carácter disposicional del concepto, cabe notar que un colectivo, para poseer la propiedad "ser un grupo de referencia" para cierto individuo en determinada circunstancia, debe, como ya se indicó, encontrarse en la situación de que "si se diera que la aspiración del individuo en esa circunstancia a afiliarse al colectivo tiene la evaluación a y la normativa del colectivo en cuestión en dicha circunstancia viene dada por los valores $\left\{v_{1}, \ldots, v_{\mathrm{k}}\right\}$, entonces se daría que el comportamiento del individuo en esa circuntancia respecto a esos valores - normativa - tiene la evaluación $a$ ". Dicho de otro modo: el que efectivamente un determinado colectivo sea grupo de referencia para un individuo en cierta circunstancia depende de que sea correcto (verdadero) un condicional subjuntivo en cuyo antecedente se establecen la condiciones de prueba y en cuyo consecuente se establece el resultado a obtener.

En lo que respecta a su carácter disposicional, casos como el del concepto "grupo de referencia" no son pocos en la ciencia. Stegmüller, por ejem- 
plo, (1970), lista diferentes instancias donde son comunes conceptos disposicionales. Nos hace ver que "muchas de las propiedades de los objetos físicos son disposicionales", que "también entre las propiedades perceptibles es frecuente encontrar disposiciones", asimismo "otra clase [de conceptos disposicionales] la constituyen las disposiciones psíquicas", además de que "numerosos conceptos de la sociología, de la antropología, de la economía y de la politología son conceptos disposicionales" y, por fin, indica "aquella clase de disposiciones que en los tratados de filosofía de la ciencia se suelen tomar como ejemplos: se trata de conceptos físicos, químicos y de otras ciencias naturales, de diverso grado de generalidad" (Stegmüller 1970, pp. 214-216 [1979, pp. 245-248]) $)^{8}$. Esta muestra de la variedad de campos donde están presentes conceptos con estas características le lleva a plantear:

Dado que en cualquier construcción sistemática de la ciencia, el número de predicados básicos debe ser pequeño para construir un sistema lo más simple posible y no perder la visión de conjunto, seguro que no se van a escoger todos los predicados disposicionales como predicados primitivos. Con ello hemos llegado a la cuestión decisiva: “¿De qué manera hay que introducir en el lenguaje de la ciencia predicados disposicionales que no sean predicados básicos primitivos?”. (Stegmüller 1970, p. $217[1979$, p. 248])

No hay que olvidar que Stegmüller con dicho pasaje se sitúa en el marco de la consideración neopositivista inicial de que el lenguaje de la ciencia tendría dos sublenguajes, uno básico observacional y otro teórico, y que a ambos sublenguajes subyacería la lógica clásica de primer orden. En tal marco el sublenguaje básico se concebía como asentado en ciertas expresiones básicas que se toman como primitivas. En lo que respecta al vocabulario descriptivo se suponía, pues, que se disponía de términos "observacionales" básicos a los que deberían reducirse, en principio vía definiciones, los otros términos científicos que tuvieran verdadera significación empírica. Dicha consideración supuso un importante problema metateórico. El caso de los términos disposicionales en dicho contexto se mostró especialmente relevante de las limitaciones de las pretensiones neopositivistas iniciales, toda vez que pese a ser aparentemente poco abstractos, y por ello próximos a términos observacionales, ni podían considerarse dando cuenta de propiedades o relaciones observables ni podían definirse mediante enunciados tales que: (i) se pueda dar cuenta de su forma lógica mediante la lógica clásica de primer orden; y (ii) sólo contengan términos observacionales básicos (términos primitivos básicos). Entre los intentos de hallar una solución en dicho marco estuvo: primero el de recurrir a las definiciones operacionales

${ }^{8}$ En adelante, lo puesto entre corchetes corresponde a la versión castellana. 
(mediante un condicional material); después el de los enunciados reductivos (Carnap, 1936).

Cabe notar que en el marco de la discusión neopositivista, y atendiendo a la distinción teórico-observacional, se plantearon dudas acerca de dónde situar los términos disposicionales, si en el ámbito de lo teórico o en el de lo observacional. Por un lado, no daban cuenta de propiedades o relaciones observables, por lo que no formaban parte de las expresiones observacionales básicas; por otro lado, parecían lo suficientemente próximos al lenguaje observacional como para pensar que cabía reducirlos (vía definiciones explicitables conforme a la lógica clásica de primer orden) a oraciones que sólo contuvieran términos observacionales básicos, y por esa vía integrarlas en el vocabulario observacional ampliado. Sin embargo, las pretensiones de reducción (vía definiciones operacionales o vía enunciados reductivos), mediante oraciones explicitables en lógica clásica de primer orden, se mostraron insatisfactorias (cf. Stegmüller, 1970, pp. 213-238 [1979, pp. 244272]). Uno de los motivos de tal inadecuación se debe al carácter subjuntivo de los condicionales que conforman las definiciones operacionales. Finalmente, en el marco de discusión neopositivista se terminó asumiendo el carácter teórico de los términos disposicionales.

En la reconstrucción aquí proporcionada, aunque no se asume una dicotomía teórico/observacional, sino una distinción relativa a cada teoría, T-teórico/T-no-teórico, parece evidente que respecto a la TGR, el término $G R U$ (o el concepto que expresa) es teórico precisamente porque su determinación depende de una ley — la que se presenta como ley de TGR - que contiene un condicional subjuntivo con las condiciones de prueba y el resultado a obtener para que un determinado colectivo sea considerado grupo de referencia para un individuo y una circunstancia. Nótese en cualquier caso que al decir esto se apunta simplemente a la peculiaridad de GRU en el marco de TGR.

\section{Conclusión}

En el trabajo, después de algunas aclaraciones preliminares y una presentación informal de la teoría, fueran presentados sus modelos potenciales, modelos, modelos potenciales parciales, sus aplicaciones intencionales y, al final, algunas observaciones acerca del carácter relacional y disposicional de la noción "grupo de referencia". De esta manera se pudo precisar qué es lo que aporta el trabajo de Merton a un mejor entendimiento de la conducta del individuo en sociedad. 
El concepto "grupo de referencia" es utilizado por Merton para explicar parte do espectro de la acción humana en sociedad. Asimismo, el tratamiento más preciso de la teoría, además de presentar los demás conceptos constituyentes de la TGR, aporta para un mejor entendimiento de su campo de aplicación. En este aspecto cabe destacar que el carácter relacional y disposicional del concepto "grupo de referencia" nos hace entender la dinámica de aplicación de la TGR.

Además, la reconstrucción supone un nuevo aporte -propiciando mejores elementos a la reflexión- a la discusión propuesta en Lorenzano \& Abreu (2010) acerca de la propuesta mertoniana referente a las teorías de alcance intermedio. Como se puede ver en el referido trabajo, es posible mostrar como el concepto de teorías de alcance intermedio de Merton puede precisarse, y entenderse mejor, si se lo relaciona, en lugar de con la concepción clásica de las teorías, con la Metateoría Estructuralista. La presente reconstrucción es un caso concreto de la discusión general presentada en aquella ocasión.

\section{Referencias bibliográficas}

Carnap, R. (1936-1937) “Testability and Meaning”, in: Philosophy of Science, 3, pp. 420-468; 4, pp. 1-40.

Kuhn, T. (1962) The Structure of Scientific Revolutions. University of Chicago Press, Chicago (trad. castellana: La Estructura de las Revoluciones Cientificas. Fondo Cultura Económica, México 1975).

Lorenzano, P.; Abreu, C. (2010) "Las teorías de alcance intermedio de Robert K. Merton y las concepciones clásica y estructuralista de las teorías”. In: Martins, R. A.; Lewowicz, L.; Ferreira, J. M. H.; Silva, C. C.; Martins, L. A. P. (eds.) (2010). Filosofia e história da ciencia no Cone Sul. Seleção de trabalhos do $6^{\circ}$ Encontro. Campinas: Associação de Filosofia e História da Ciência do Cone Sul (AFHIC), pp. 482-492.

Merton, R. K. (1949-1957) Social Theory and Social Structure, engarged edition. New York: The Free Press, 1968 (trad. cast.: Teoría y Estructura Sociales. 4. ed. México: Fondo de Cultura Económica, 2002).

Moulines, C. U. (1986) "Redes teóricas". In: Rolleri, J. L. Estructura y desarrollo de las teorías científicas. México: UNAM, pp. 299-326.

Stegmüller, W. (1970) Theorie und Erfahrung. Springer, Berlín-Heidelberg, (trad.. cast.: Teoría y Experiencia. Ariel, Barcelona, 1979). 